\title{
Psicooncología
}

ISSN: $1696-7240$

\section{Ajuste psicológico en niños brasileños con cáncer y en sus padres o cuidadores}

Autora: Amanda Muglia Wechsler

Tipo de trabajo: Tesis Doctoral

Directoras: María del Carmen Bragado Álvarez y María José Hernández Lloreda

Universidad: Universidad Complutense de Madrid, Departamento de Personalidad, Evaluación y Tratamientos Psicológicos (Psicología Clínica)

Fecha de lectura: 22/01/2016

E-mail: amanda_wechsler@yahoo.com.br

Aunque el cáncer sigue siendo la segunda causa de muerte de niños y adolescentes de todo el mundo, en las últimas décadas la tasa de supervivencia ha ido en aumento gracias a los avances producidos en el tratamiento de esta enfermedad. En Brasil, cerca de 9.000 niños entre 0 y 19 años son diagnosticados con cáncer cada año, de los cuales alrededor del 70\% sobreviven diez años después del diagnóstico. Esta realidad ha propiciado el interés por conocer el impacto psicológico de la enfermedad a largo plazo. Sin embargo, mientras que la producción científica sobre el ajuste psicológico de los supervivientes es relativamente extensa, la investigación de este aspecto en los niños que sufren una recaída es mucho más limitada, y en ambos casos es casi inexistente en el contexto Brasileño.

Objetivos: Estudiar el grado de ajuste psicológico de niños brasileños aquejados de cáncer (en recaída y supervivientes) y de sus padres o cuidadores, comparados con un grupo control de niños sanos ( la relación existente entre una serie de variables sociodemográficas, médicas, clima familiar y apoyo social recibido por los cuidadores (mencionadas en la investigación previa) y las medidas de ajuste empleadas en esta investigación.

Sujetos: En este estudio participaron un total de 180 niños/adolescentes de 6 a 14 años (52\% mujeres) distribuidos en tres grupos: 1) Recaída, integrado por 31 niños y 32 cuidadores; 2) Supervivientes (sin recidivas), formado 78 niños y 75 cuidadores; y 3) Control, integrado por 71 niños sin historia conocida de cáncer y 76.

Metodología: Para evaluar el grado de ajuste se emplearon los siguientes instrumentos de medida; con los niños: el Cuestionario de Conducta Infantil (BASC), la Escala de Ansiedad Manifiesta (RCMAS) y la Escala de Autoconcepto de PiersHarris; y con los padres o cuidadores: el BASC (versión para padres), los inventarios de ansiedad y depresión de Beck, el Cuestionario de Síntomas de Derogatis (SCL90-R), el Cuestionario de Apoyo Social de Norbeck y la Escala de Clima Social en la Familia. 
Resultados: Los resultados más destacables de este estudio indican que los dos grupos aquejados de cáncer (en situación de recidiva y supervivientes) no solo no presentaban más problemas psicológicos que el grupo control, sino que, además, mostraron mejores resultados que sus pares "sanos" en distintas variables. Concretamente, los pacientes en recidiva manifestaron significativamente menos conductas atípicas y más habilidades adaptativas y sociales que los controles, mientras que los supervivientes mostraron menos ansiedad, más liderazgo y más habilidades adaptativas y sociales que los integrantes del grupo control. Sin embargo, los cuidadores de niños en situación de recaída (pero no los cuidadores de supervivientes) mostraron niveles significativamente más elevados de depresión, obsesión-compulsión e intensidad de los síntomas emocionales que los otros dos grupos (supervivientes y control). También se encontró una asociación significativa entre una mayor cohesión familiar y menos problemas psicopatológicos en los niños y sus respectivos cuidadores, pero dicha asociación no se confirmó para el apoyo social. Además, se encontraron relaciones significativas entre un menor grado de ajuste y el género (niños varones y mujeres adolescentes), la menor edad de los niños, el estado civil de los padres (sin pareja) y un bajo nivel socioeconómico y educativo. Por otro lado, un mayor número de recidivas y más tiempo en tratamiento oncológico se encontraron significativamente asociados con un peor ajuste psicológico de los niños en recidiva o supervivientes y de sus cuidadores.

Conclusiones: Se puede concluir que los niños con cáncer presentan un ajuste psicológico adecuado, tanto en la situación de recidiva como en el postratamiento. Sin embargo, los cuidadores de niños en recidiva podrían requerir atención especializada, puesto que manifiestan más dificultades de ajuste que los del grupo de supervivientes o los del control. Se destaca también el posible efecto protector de la cohesión familiar, la educación parental, la mayor edad del niño y una buena renta familiar sobre el ajuste psicológico de niños con cáncer y de sus cuidadores. 